\title{
Modélisation des plasmas produits par décharge électrique
}

\author{
I. Pérès et L. C. Pitchford \\ Laboratoire des Décharges dans les Gaz, C.P.A.T., 118 route de Narbonne, \\ 31062 Toulouse Cedex, France
}

\begin{abstract}
:
An overview of the modeling of non-thermal plasmas produced in electric discharges is presented. Two examples are given to illustrate the state of the art; a collisionnalradiative model of a small diameter, argon-mercury discharge for lighting application, and a self-consistent electrical model of pseudo-spark transient hollow cathode.
\end{abstract}

Introduction

De façon schématique, on peut représenter une décharge électrique comme un système composé de trois éléments : le volume gazeux dans lequel se produit la décharge, les électrodes entre lesquelles on applique un champ électrique, ce qui permet le passage d'un courant dans le gaz, et l'enceinte qui englobe le système. De même que dans une lampe à filament le courant qui parcourt le filament chauffe celui-ci jusqu'à incandescence, lorsque le courant traverse le gaz, il dépose une partie de son énergie en excitant, dissociant, et ionisant les molécules ou atomes du gaz. Si la pression du gaz est élevée, de l'ordre d'une atmosphère, et si le courant est maintenu pendant un temps suffisamment long, le plasma produit est thermique, la température du gaz est alors égale à la température des électrons [1]. Pour des pressions de gaz plus basses, la température électronique est beaucoup plus importante que celle des particules lourdes, et les populations des états excités sont tout à fait non-thermiques [1]. Nous nous limiterons ici au cas de ces décharges non-thermiques.

Les électrons jouent un rôle central dans le bilan d'énergie de la décharge. Plus légers que les ions, ils suivent plus facilement les variations du champ électrique, et sont l'agent principal du transfert d'énergie entre le champ électrique et les neutres, par les collisions qu'ils effectuent avec ceux-ci. L'énergie transmise aux neutres par les électrons est ensuite évacuée du système par émission de rayonnement et transfert de chaleur sur les parois de l'enceinte et les électrodes. Les électrodes interviennent aussi activement dans le développement de la décharge en produisant des électrons secondaires. Ceux-ci sont émis sous l'effet du bombardement des ions produits dans la décharge lorsque les électrodes sont froides, ou par émission thermoionique si le transfert d'énergie est suffisant pour chauffer les électrodes. Dans les exemples qui suivent, nous ne nous intéressons qu'à des décharges à cathode froide

La difficulté de développer un modèle complet de décharge électrique provient du couplage qui existe entre la cinétique des particules chargées (électrons et ions), celle des particules lourdes (neutres dans l'état fondamental ou dans des états excités) et la distribution de champ électrique. Du fait de la complexité du problème, les modèles autocohérents complets ne sont pas utilisables systématiquement, et l'un ou l'autre des aspects du couplage doit être privilégié. Cette simplification est généralement rendue possible par les caractéristiques physique des systèmes étudiés [2]. 
Après une présentation des étapes nécessaires au développement d'un modèle physique, nous illustrerons dans ce qui suit l'état de la modélisation des décharges électriques par deux exemples de modèles autocohérents :

- les modèles collisionnels-radiatifs [3], qui sont utilisés lorsque les interactions entre les neutres et les électrons sont prépondérantes. Lorsque dans une décharge le degré d'excitation du gaz devient important, la mobilité des électrons et les taux de transfert d'énergie dans le gaz changent, ce qui modifie le transfert d'énergie entre les électrons et les neutres.

- les modèles électriques [2], qui permettent d'étudier le couplage entre le champ électrique et les particules chargées : les particules se déplaçant dans un champ électrique qui est la superposition du champ aopliqué et du champ de charge d'espace, dû aux particules chargées en mouvement, le champ électrique se modifie à mesure que les charges se déplacent vers les électrodes.

\section{Modèle physique}

Pour être fidèle à la réalité physique, un modèle doit reproduire aussi finement que possible les phénomènes physiques mis en jeu dans le système étudié. La tâche du modélisateur est donc tout d'abord d'identifier les caractéristiques principales du problème physique, et de les formuler mathématiquement. Du fait de la complexité des systèmes étudiés, cette représentation mathématique est liée au choix d'approximations et d'hypothèses qui rendent le problème soluble.

La formulation du problème fait apparaître le besoin de données permettant de quantifier les processus physiques de base. La recherche de ces données devient un point essentiel pour la modélisation des systèmes complexes dans lesquels un grand nombre d'espèces interagissent entre elles selon des procédés multiples.

La troisième étape fondamentale du développement du modèle, est le choix, ou le développement, de méthodes numériques qui permettent la résolution des équations choisies, en tirant parti au mieux des spécificités du problème, et des moyens de calcul disponibles.

Le problème central reste cependant la formulation mathématique du problème physique. Les éléments responsables directement du développement de la décharge sont les particules chargées, les neutres et le champ électrique. La base d'un modèle général est donc la résolution simultané des équations de Maxwell, pour les champs électrique et magnétique, de l'équation de Boltzmann, écrite pour chacune des espèces produites dans la décharge, et de l'équation de transfert du rayonnement. On obtient alors, les distributions đu champ électrique, les fonctions de distribution des vitesses des particules en fonction de la position dans la décharge et du temps, dont on déduit toutes les autres grandeurs d'intérêt. En fait, un tel modèle serait trop "lourd" pour une utilisation systématique, mais les caractéristiques de décharge permettent de faire un certain nombre d'approximations :

- Sauf pour les décharges excitées par des ondes de très haute fréquence, les équations de Maxwell peuvent être restreintes à l'équation de Poisson, qui donne la distribution de potentiel entre les électrodes. Même sous cette forme simplifiée, le calcul du champ électrique reste délicat lorsque l'on modélise une géométrie complexe, et le choix d'une méthode numérique adéquate est important.

- L'équation de transfert du rayonnement peut être négligée, si le rayonnement est optiquement mince.

- L'évolution des densités de neutres est décrite par une équation de continuité, si la décharge est non-thermique, et si le flux hydrodynamique peut être négligé. La résolution de 
cette équation nécessite néanmoins la connaissance des taux de production des différentes espèces, ce qui peut représenter un grand nombre de données.

- En ce qui concerne les particules chargées, la résolution numérique de l'équation de Boltzmann peut être évitée si l'on choisit de représenter les propriétés de ces particules par trois grandeurs moyennes, par exemple, la densité, la vitesse moyenne et l'énergie moyenne. Les trois premiers moments de l'équation de Boltzmann dans l'espace des vitesses fournissent alors les équations représentant l'évolution spatio-temporelle de ces trois grandeurs moyennes : équation de continuité, équation de transfert de quantité de mouvement et équation d'énergie. Les modèles basés sur ce principe sont dits modèles fluides.

La résolution de ce système d'équations pour chacune des espèces chargées est presque aussi difficile que la résolution directe de l'équation de Boltzmann, et d'autres hypothèses simplificatrices sont nécessaires. Par exemple,

- représentation du transport des particules par une équation de transport sous la forme de la somme d'un terme de dérive et d'un terme de diffusion,

- approximation du champ électrique local ou de l'énergie moyenne locale pour la détermination des paramètres macroscopiques de transport, et des fréquences de collision,...

- répartition des électrons en deux groupes, pour différencier le comportement des électrons "rapides", ayant gagné une énergie importante dans les zones de fort champ électrique, et qui viennent déposer cette énergie dans le plasma , et celui des électrons "lents", de faible énergie, qui composent le corps du plasma et peuvent être traités par l'approximation du champ électrique local.

Il existe cependant des situations où la résolution de l'équation de Boltzmann reste préférable pour le calcul des fréquences de collision. Dans ce cas, on utilise des méthodes particulaires, Monte Carlo - Particle In Cell, basées sur le suivi des trajectoires d'un grand nombre de particules dans l'espace des phases. Le champ électrique étant calculé par résolution de l'équation de Poisson. Un traitement statistique permet d'obtenir les fonctions de distribution en énergie des particules, et d'en déduire les fréquences de collision correspondant au profil de champ donné.

Les avantages des modèles fluides et ceux des modèles particulaires étant complémentaires, une nouvelle génération de modèles est apparue, basée sur le couplage d'une description "fluide" du transport des particules avec une détermination "particulaire" des paramètres de transport et des fréquences de collision. L'utilisation systématique de ces modèles hybrides est rendue possible par l'évolution récente des moyens de calcul.

De ce qui précède, on peut conclure que le développement du modèle est directement lié à l'identification des éléments prépondérants dans le couplage particules chargées / particules lourdes / champ électrique. Fort heureusement, les situations de décharge dans lesquelles les trois aspects du couplage ont une importance égale sont rares, et il est souvent possible de privilégier l'étude de l'un ou l'autre de ces aspects du couplage par des approximations convenables. Cependant, il reste essentiel de déterminer le domaine de validité du modèle, limitế par les approximations choisies, et ces limites de validité ne peuvent être précisées que par la comparaison des résultats obtenus avec des résultats expérimentaux ou d'autres modèles.

\section{Exemples d'applications des modèles de décharges électriques}

Nous illustrons ici deux aspects différents de la modélisation des décharges. Dans le premier exemple qui sera développé, le modèle permet de quantifier le transfert d'énergie entre 
les électrons et les neutres, et entre différentes espèces excitées, dans une situation où le champ électrique peut être déterminé directement. Le second exemple démontre l'utilité de la modélisation pour l'identification d'une séquence d'événements physiques quand plusieurs phénomènes fortement couplés entrent en jeu.

\section{$a$ - Modèle collisionnel-radiatif}

Une simplification importante du modèle est possible quand il n'est pas nécessaire de calculer de façon auto-cohérente le champ électrique de charge d'espace. C'est le cas par exemple lorsque les densités de particules crées dans la décharge ne suffisent pas à modifier le champ électrique appliqué. C'est aussi le cas lorsque le champ électrique est spatiallement uniforme. Par exemple, dans la colonne positive d'une décharge, région comprise entre la fin de la région de gaine cathodique et l'anode, les caractéristiques électriques et spectrales sont uniformes selon l'axe de la décharge. Dans ces conditions, la valeur du champ axial à l'état stationnaire peut être évaluée, car c'est le champ qui donne la température électronique nécessaire pour compenser par ionisation les pertes de particules chargées dues à l'attachement, la recombinaison ou la diffusion vers les parois. Par contre, les mécanismes de transfert d'énergie entre les particules chargées et les différentes espèces excitées produites peuvent devenir très complexes et nécessitent une étude détaillée des différents processus possibles.

Le cas de la lampe fluorescente [4] est sans doute l'exemple le plus classique de colonne positive. La décharge est produite dans un mélange de mercure (à une pression de quelques mtorr) et d'argon (à quelques torr), par application d'une tension de l'ordre de quelques centaines de volts. Dans ces lampes, la raie de résonance à $254 \mathrm{~nm}$ du mercure est produite avec une efficacité apprcchant $65 \%$ dans les lampes optimisées. Une tendance actuelle dans l'industrie est le développement de lampes fluorescentes compactes, qui nécessitent une production de photons à $254 \mathrm{~nm}$ par unité de longueur plus importante que dans les lampes classiques. Le problème est de réussir à forcer la décharge à absorber plus de puissance par unité de longueur, alors que, au-dessus d'une certaine puissance, on observe que le gaz à tendance à thermaliser, et que, en conséquence, l'efficacité de rayonnement diminue.

Cette thermalisation du gaz étant liée à une baisse de la température électronique dans la décharge, une solution évidente est d'augmenter les pertes électroniques dans la décharge. En effet, la production de charges doit alors augmenter, pour que l'équilibre entre gains et pertes reste vérifié dans la colonne, ce qui maintient une température électronique élevée. L'augmentation des pertes peut être obtenue par une diminution du diamètre du tube de décharge, car le principal mécanisme de perte provient de la diffusion vers les parois. G. Zissis et al. [5] ont publié récemment un modèle détaillé du bilan d'énergie dans la colonne positive d'une décharge argon/mercure, pour l'évaluation de la production de photons à $254 \mathrm{~nm}$ dans des tubes de décharge de petit diamètre (inférieur à $15 \mathrm{~mm}$ ). Ce modele est un exemple de l'état de l'art de la modélisation de la colonne positive. Il permet de calculer les densités de population du niveau fondamental du mercure et de ses 5 premiers niveaux excités, ainsi que les températures et densités des électrons et du gaz rare. Les principaux processus collisionnels sont pris en compte : collisions élastiques, collisions inélastiques électron-atome et atomeatome, incluant les divers processus d'excitation, d'ionisation directe et par étape, et collisions super-élastiques. Les valeurs du champ électrique dans la colonne et de la puissance émise sous forme de rayonnement y sont aussi déterminées. Cette complexité du modèle permet d'avancer dans la compréhension des phénomènes physiques se produisant dans la colonne 
positive. En particulier, l'un des résultats de ce modèle est la mise en évidence du fait que, bien qu'il soit possible d'augmenter de manière significative la puissance absorbée par unité de longueur en diminuant le diamètre du tube, le nombre total de photons UV générés par unité de longueur diminue, du fait de la diminution du volume de décharge. Il est intéressant de noter aussi que dans les conditions optimum, le bilan d'énergie dans une décharge classique (quelques centimètres de diamètre) et dans une décharge de faible diamètre est le même. De ce fait, dans les deux cas, environ $65 \%$ de la puissance absorbée conduit à la production de photons UV.

\section{b - Modèle électrique}

Parmi les nombreuses applications des modèles électriques, nous avons choisi d'illustrer ce propos par le cas des décharges pseudo-sparks [6]. C'est en effet un cas typique de décharge essentiellement contrôlée par l'évolution spatio-temporelle du champ électrique de charge d'espace. Le principe général des pseudo-sparks est simple, cependant les différents étapes du développement de ces décharges n'ont pu être clarifiées que grâce à la modélisation. Nous avons utilisé pour cela un modèle hybride fluide-particulaire, dépendant du temps, tridimensionnel à symétrie cylindrique [7].

L'application principale des décharges pseudo-sparks est la commutation rapide de courants intenses. Des montées de courant de l'ordre de $10^{12} \mathrm{~A} / \mathrm{cm}^{2}$ ont été observées [6], pour des tensions appliquées de l'ordre de quelques dizaines de kilovolts, dans plusieurs gaz d'usage courant. Ils sont aussi utilisés comme sources de faisceaux d'électrons et de rayons $\mathrm{X}$ [8].

La configuration la plus simple dans laquelle un pseudo-spark apparaît est une cathode creuse rectangulaire percée d'un trou et faisant face à une anode plane. Le produit de la pression et de la distance interélectrode (inférieur à 1 torr.cm dans l'hélium) est tel que, d'après la courbe de Paschen, le claquage se produit entre l'anode et la face interne de la cathode. Le déclenchement de la décharge est assuré de manière externe par injection de charges, éclairement UV ou application d'un champ magnétique. Ce déclenchement crée des paires électrons-ions dans la cavité de la cathode creuse. Lorsqu'une tension suffisante est appliquée entre les électrodes, les électrons initiaux et les électrons seconđaires libérés à la cathode par bombardement ionique donnent naissance à des avalanches successives, par ionisation du gaz, et contribuent à la formation d'une charge d'espace ionique importante. Pour certaines combinaisons de la tension appliquée, de la pression du gaz, et de la géométrie, la charge d'espace ionique peut finir par produire un champ de charge d'espace suffisant pour déformer le champ électrique initial. Un plasma se forme alors, car les électrons sont ralentis dans la zone de forte charge d'espace ionique. Pour des tensions relativement basses, la formation du plasma commence près de l'anode. Puis, le plasma se dilate jusqu'à pénétrer dans la cathode creuse, emportant avec lui le potentiel d'anode. Dans cette configuration de champ, la multiplication électronique est extrêmement efficace pour deux raisons. Premièrement, l'effet de confinement de la géométrie force les électrons à osciller dans la cavité et à y déposer une grande partie de leur énergie. Deuxièmement, les électrons secondaires produits par ionisation đu gaz sont aussi créés dans des régions de champ électrique élevé où ils peuvent rapidement gagner une énergie importante. De ce fait, contrairement au cas des décharges en géométrie plan parallèle où les électrons sont créés dans des régions de faible champ, les secondaires et leurs propres générations contribuent aussi à l'augmentation de l'ionisation. Une montée brusque du courant est observée au moment où le plasma entre dans la cathode creuse. 
Cette suite d'événements est illustrée sur la figure 1, qui montre des résultats obtenus dans l'hélium à 0.5 torr et pour une tension appliquée de $2 \mathrm{kV}$. Les contours équipotentiels calculés par le modèle y sont représentés à quatre instants différents du développement de la décharge. Dans le premier cas (fig. $1 \mathrm{a}, \mathrm{t}=6 \mathrm{~ns}$ ), le champ électrique est essentiellement le champ géométrique appliqué, sans perturbation de la charge d'espace. Après 744 ns (fig. 1b), un plasma s'est formé près de l'anode, et à 844 ns (fig. 1c), l'extension spatiale du plasma a augmenté et celui-ci commence à pénétrer dans la cathode creuse. C'est à 844 ns que la multiplication atteint son maximum et une brusque augmentation du courant se produit. Finalement (fig. 1d, $t=1020 \mathrm{~ns}$ ), le plasma remplit le volume de la cathode, et les régions de fort champ électrique, les gaines, sont compressées contre les parois internes de la cavité. A ce stade, l'émission de champ ou l'émission thermoionique vont pouvoir commencer à jouer un rôle, car la situation n'est pas stationnaire et les gaines continuent à se contracter. L'extension du modèle, incluant ces autres mécanismes d'émission électronique est en cours actuellement, ce qui permettra d'étudier l'évolution ultérieure de la décharge.
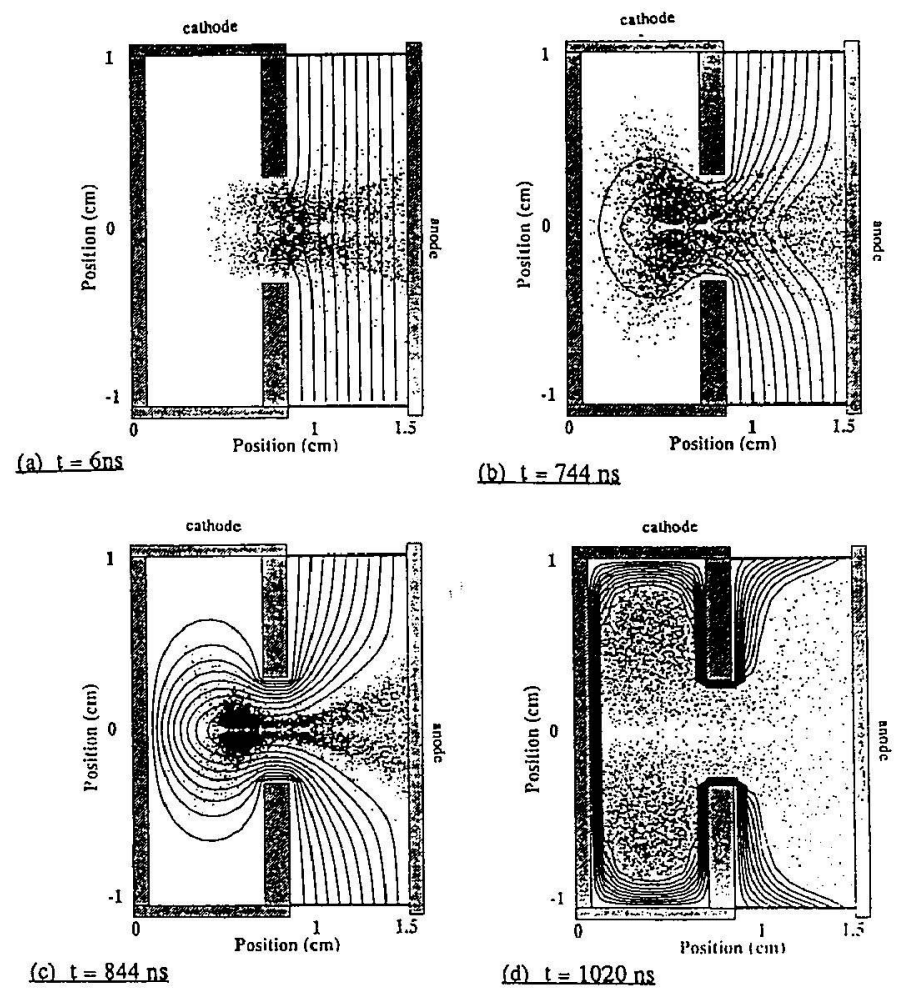

Figure 1 : Contours équipotentiels à quatre instants du développement de la décharge. [ Les points superposés représentent les emplacements où se sont produits des événements d'ionisation ]. Hélium, pression : 0.5 torr, potentiel d'anode : $2 \mathrm{kV}$

\section{Conclusion}

Pour conclure, nous ferons quelques commentaires sur l'état de l'art de la modélisation des plasmas non thermiques produits par décharge électrique [9]. 
Bien que les éléments contrôlant l'évolution d'une décharge soient bien connus, il n'est pas réaliste de vouloir développer un modèle complet tenant compte de tous ces éléments et de leurs interactions. De ce fait, les modèles, basés sur des approximations qui permettent de rendre le problème physique soluble numériquement, ont un domaine de validité limité.

L'évaluation continuelle, par la comparaison avec des résultats expérimentaux, est un point essentiel pour le développement d'un modèle, permettant d'en vérifier la validité et d'apporter des améliorations. Le propos n'étant pas ici de rentrer dans tous les détails des deux modèles cités en exemple, de telles comparaisons n'ont pas été présentées. Cependant, dans ces deux cas, l'accord avec l'expérience est excellent.

Le domaine d'application des plasmas produits par décharge électrique est vaste, et le modèle est d'autant plus efficace qu'il est bien adapté à l'application étudiée. Les modèles collisionnels-radiatifs prennent en compte les interactions entre les particules chargées et les neutres, et sont seulement limités par les données disponibles pour l'identification des nombreux processus d'échange d'énergie entre les neutres et les électrons. Les modèles électriques reproduisent les interactions entre les particules chargées et le champ électrique. Des modèles performants existent en 1 et 2 dimensions (en géométrie cylindrique ou rectangulaire). Les modèles en 3 dimensions, en géométrie non symétrique, ne sont pas encore pratiques (en termes de temps de calcul). Le couplage d'un modèle collisionnel radiatif avec un modèle électrique de la décharge est possible, et a été réalisé pour quelques systèmes spécifiques. Là encore, la principale limitation au développement de ces modèles vient des données nécessaires. Les exemples présentés ici (colonne positive d'une lampe fluorescente et décharge pseudo-spark), ont été choisis parce qu'ils sont typiques des modèles présentés. Un autre exemple de modélisation sera développé au cours de ce colloque dans le domaine des lasers à excimères [10].

Enfin, avec chaque évolution de la technologie des ordinateurs, des modèles de complexité croissante deviennent utilisables. De prochains progrès dans l'état de l'art de la modélisation sont à attendre, avec la généralisation des machines à processeurs parallèles.

\section{Références}

[1] De nombreux articles et ouvrages de synthèse ont été consacrés aux décharges électriques, parmi les plus intéressants citons, Von Engel A., "Ionized Gases", Clarendon Press, Oxford (1965).

Druyvesteyn M.J. et Penning F.M., "The mechanism of electrical discharges in gases of low pressure", Reviews of Modern Physics, 12(2), 87 (1940).

[2] Boeuf J.P., Belenguer Ph., Pitchford L.C. et Pérès I., $8^{\text {th }}$ International Colloquium on Plasma Processes, Antibes, France, Juin 1991, Short Courses on Plasma Chemical Deposition and Etching, Eds GR 57 (CNRS) et Société Française du vide.

[3] Bates D.R., Kingston AE et McWhirter R.W.P., Part I, Proc. Roy. Soc. A, 267, 297 (1962); Part II, Proc. Roy. Soc. A, 270, 155 (1962).

[4] Waymouth J.F., "Electric Discharge Lamps", MTT Press, Cambridge (1971).

[5] Zissis G., Bénétruy P. et Bernat I., Phys. Rev. A, 45(2), 1135 (1992).

[6] Gundersen M.A. et Schaefer G., Eds, "The Physics and Applications of Pseudosparks". New York: Plenum (1990).

[7] Boeuf J.P.et Pitchford L.C., IEEE Trans. on Plasma Sci., 19(2), 286 (1991).

[8] Pigache D., ce volume.

[9] Voir aussi IEEE Trans. on Plasma Sci., Vol 19 (1991), volume consacré à la modélisation des plasmas non-thermiques.

[10] Bretagne J., ce volume. 